\title{
Sea Turtle Identification and Viewing Guidelines ${ }^{1}$
}

\section{Lindsay Nester and William M. Giuliano ${ }^{2}$}

A walk down a Florida beach may result in an unexpected encounter with one of nature's oldest reptiles, the sea turtle (Figure 1). From early spring through fall, female sea turtles travel across the sandy beach to deposit their eggs beyond the surf line in the warm sand. A sick, injured, or dead turtle may also be found on the shoreline during any season of the year. An observer may have several questions about this experience: Which agency should be called if the turtle appears to need help? What are appropriate or legal interactions with a sea turtle? What type of sea turtle is it? Does this sea turtle need assistance?

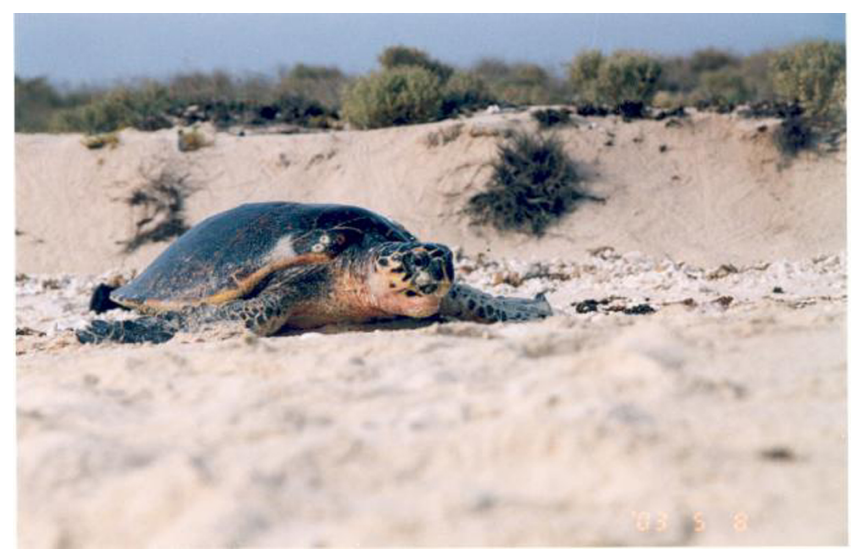

Figure 1. A walk down a Florida beach may result in an unexpected encounter with one of nature's oldest reptiles, the sea turtle. Credits: A. Mobaraki (2005).

\section{Guidelines for Observing Nesting Turtles}

What should an individual do when encountering a sea turtle? In the southeastern United States, sea turtles swim to the shore for two reasons. The main reason a sea turtle would leave the comfort zone of the ocean and come ashore is to lay eggs. Female sea turtles, which can be distinguished from males by presence of a much smaller tail, are often seen actively moving up the beach to nest. They are very alert to what is going on around them and their path should not be blocked. Any disturbance can cause a sea turtle to abandon its nesting attempt and return to the water. It is important that observers stay back at least 50 feet, remain quiet, and keep all lights off. Do not take flash pictures of a nesting turtle because the flash from a camera could disorient a sea turtle or cause it to abandon nesting. It may appear that a sea turtle is struggling up the beach or back to the water, but it is important that sea turtles not be assisted in any manner. Sea turtles are slow moving and may often pause, but this type of movement is normal behavior. If a sea turtle is in need of assistance (hit by a car, on a highway, trapped in a beach feature, etc.) do not attempt to help it without calling the Florida Fish and Wildlife Conservation Commission (FWC)

1. This document is WEC 209 and one of a series of the Department of Wildlife Ecology and Conservation, Florida Cooperative Extension Service, Institute of Food and Agricultural Sciences (IFAS), University of Florida. First published: May 2006. Please visit the EDIS Web site at http://edis.ifas.ufl.edu for more publications.

2. Lindsay Nester is a Graduate Student and William M. Giuliano is an Assistant Professor and Wildlife Extension Specialist; Department of Wildlife Ecology and Conservation, Cooperative Extension Service, Institute of Food and Agricultural Sciences, University of Florida, Gainesville, FL 32611.

The Institute of Food and Agricultural Sciences (IFAS) is an Equal Opportunity Institution authorized to provide research, educational information and other services only to individuals and institutions that function with non-discrimination with respect to race, creed, color, religion, age, disability, sex, sexual orientation, marital status, national origin, political opinions or affiliations. U.S. Department of Agriculture, Cooperative Extension Service, University of Florida, IFAS, Florida A. \& M. University Cooperative Extension Program, and Boards of County Commissioners Cooperating. Larry Arrington, Dean 
for assistance. The 24-hour number is

1-888-404-3922. This agency can dispatch a turtle biologist to the area and give instructions on how to help the sea turtle.

If you would like to experience the fascinating event of watching a sea turtle nest, there are many opportunities to do so. Numerous organizations, which are permitted by the State of Florida, conduct public turtle watches each year at locations throughout the state. These programs are given by trained and knowledgeable people that ensure the participants get to experience this awesome natural event without negatively impacting the nesting turtle. Contact the Sea Turtle Survival League at 352-373-6441 for the name of an organization near you that offers public turtle watch programs.

\section{Guidelines for Observing Nest Hatching}

If you encounter a hatching nest, follow the same guidelines for observing a nesting turtle--stay back from the hatchlings (baby sea turtles) and turn off all lights (Figure 2). Light can disorient hatchlings and cause them to move away from the ocean, where they may get tired and die before reaching the ocean. It may be tempting to help hatchlings reach the sea, but they must make this journey on their own. No one knows definitively how turtles learn to navigate, but it is thought that the walk down to the ocean is important for sea turtle hatchlings to learn a sense of direction. Without a sense of direction, a sea turtle will not be able to find feeding grounds, mating grounds, or its nesting area. It is also essential not to stand near the tide line in the area where hatchlings are entering the ocean. The hatchlings often wash back in the surf and could end up behind an individual standing along the shore--it would be possible to step on the hatchlings without even knowing it!

\section{Guidelines for Dealing with Stranded Turtles}

Another reason you may encounter a sea turtle on a Florida beach is because it has washed or came ashore due to an injury or illness. A sick or injured sea turtle will often not move far above the tide line.

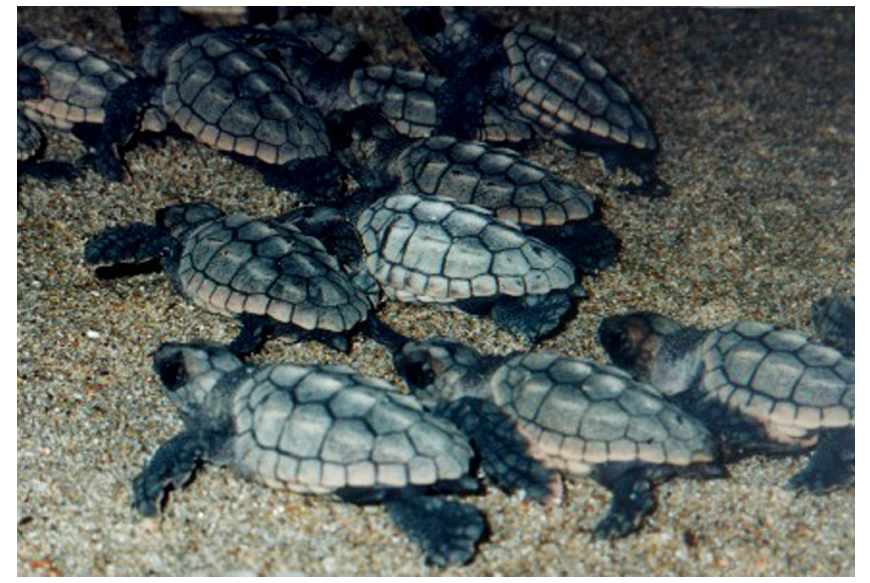

Figure 2. If you encounter a hatching nest, stay back from the hatchlings (baby sea turtles) and turn off all lights. Credits: L. Avens (2003).

Sick turtles will be lethargic, and it may be difficult to distinguish whether a turtle is alive or dead. If a live, stranded turtle (on the beach for some reason other than nesting) is found on the beach, it is critical not to touch the turtle. Sea turtles, like humans, may have injuries that can be made worse by handling. Although sea turtles are not usually aggressive, they have been known to bite people, if scared, sick, or harassed. A bite, especially from a loggerhead, can be very dangerous, with the possibility of losing fingers. If you find a stranded turtle, immediately call the FWC's 24-hour hotline 1-888-404-3922, and be prepared with the following information: species, approximate size, location, any visible injuries, and best access point to the sea turtle. Follow the instructions given very closely because the safety of the observer and turtle's survival depend on it.

If a dead, stranded sea turtle is found, call the FWC's 24-hour number with the information stated above. If the turtle is marked with spray paint, there is no need to report the turtle because it has already been found and reported. Remember that sea turtles are protected under the Endangered Species Act, which provides federal protection for all endangered species and their habitats, and to take all or part of a turtle, whether alive or dead, is a felony offense.

\section{Identification}

Due to the threatened and endangered status of all sea turtles in the United States, it is important for an observer to be aware of proper viewing procedures. It is also useful for species information to 
be given along with the location when calling for assistance. A basic knowledge of sea turtles likely to be seen on Florida's beaches would be helpful when sharing with the FWC any information about a sighting. The two species most commonly seen in Florida are the green turtle and the loggerhead turtle. Some other types of sea turtles, the leatherback, hawksbill, and Kemps ridley, are much less common in the southeastern U.S. and far less likely to be observed.

The leatherback is a very unique type of sea turtle and easiest to identify. An adult weight of up to 1,000 pounds makes the leatherback the largest species of sea turtle. However, size alone cannot be used to identify sea turtles, as turtles are found at different life-stages. The leatherback gets its name from its shell (carapace), which appears "leathery" and is smooth with seven ridges (keels) going down its back (Figure 3). The shell does not have a plated (scutes) appearance, like the green turtle (Figure 4). The coloration of the skin and shell is black with white spotting. When a leatherback is on land, the full weight of its massive body may cause the skin to turn pink, as the turtle cools its body by pumping blood near the skin's surface.

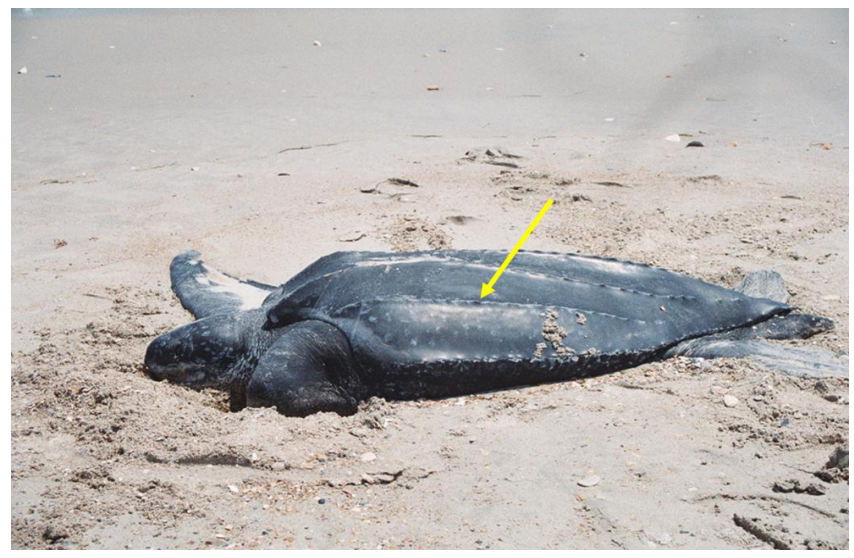

Figure 3. Leatherback sea turtle (note the "leathery" shell that is smooth with seven ridges going down its back). Credits: R. Scarpino (2005).

The green turtle is the largest sea turtle commonly found on Florida's beaches. It weighs in at 300 pounds as an adult. The green turtle, like most other sea turtles in the world, has a shell in the traditional sense. This shell is hard and segmented into scutes. The shape of the shell is oval with smooth edges. There are four pairs of scutes along the outer edge of a green turtle's shell (Figure 4). A brown, grayish, or greenish sunburst pattern covers the entire shell. The green turtle's head is small and round.

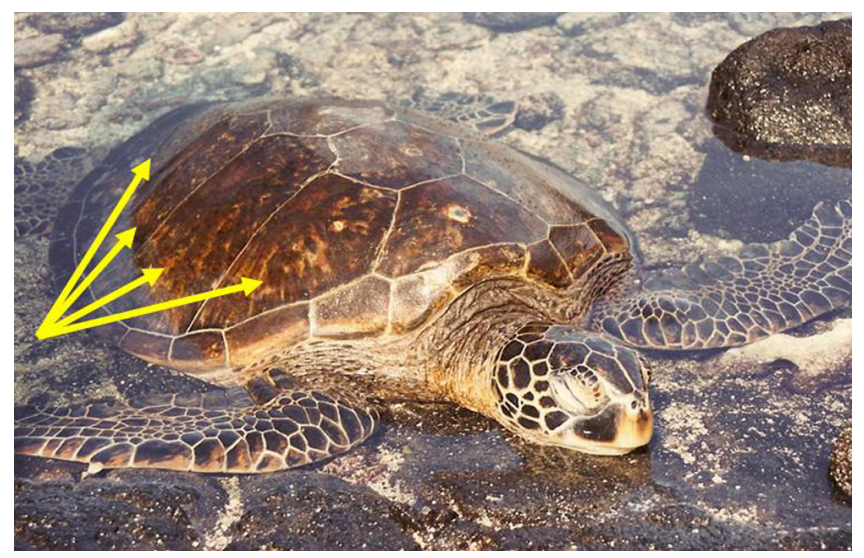

Figure 4. Green turtle (note the four pairs of scutes along the outer edge of the shell). Credits: L. Nester. (2005).

A full-grown loggerhead sea turtle is typically a little smaller than a green turtle. The loggerhead's adult weight is only about 250 pounds. A heart-shaped shell with five pairs of scutes running along the outer edge covers the loggerhead's body (Figure 5). The most distinguishing feature of the loggerhead is its massive head. The mouth comes to a point, creating a triangular-shaped head. The shell is a dull, brownish-red color and the belly (plastron) is yellow.

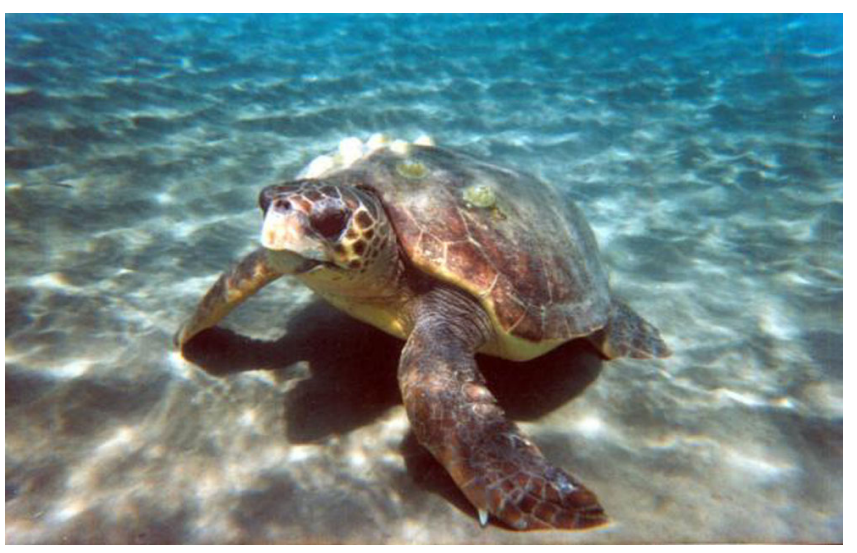

Figure 5. Loggerhead turtle (note the large head and heart-shaped shell with five pairs of scutes along the outer edge). Credits: A.F. Rees. (2003).

The hawksbill is most common in tropical waters but is occasionally seen in Florida. On average, a hawksbill weighs 125 pounds at maturity. The most distinctive feature is the hawksbill's bird-like, beak-shaped head. A beautiful and brightly colored shell is another of the hawksbill's 
distinguishing features. Like the green turtle, there are four pairs of scutes along the outer edge of the hawksbill's shell. However, these scutes overlap, making them hard to distinguish. Before protection under the Endangered Species Act, hawksbills were hunted to near extinction for the colorful shell (Figure 6).
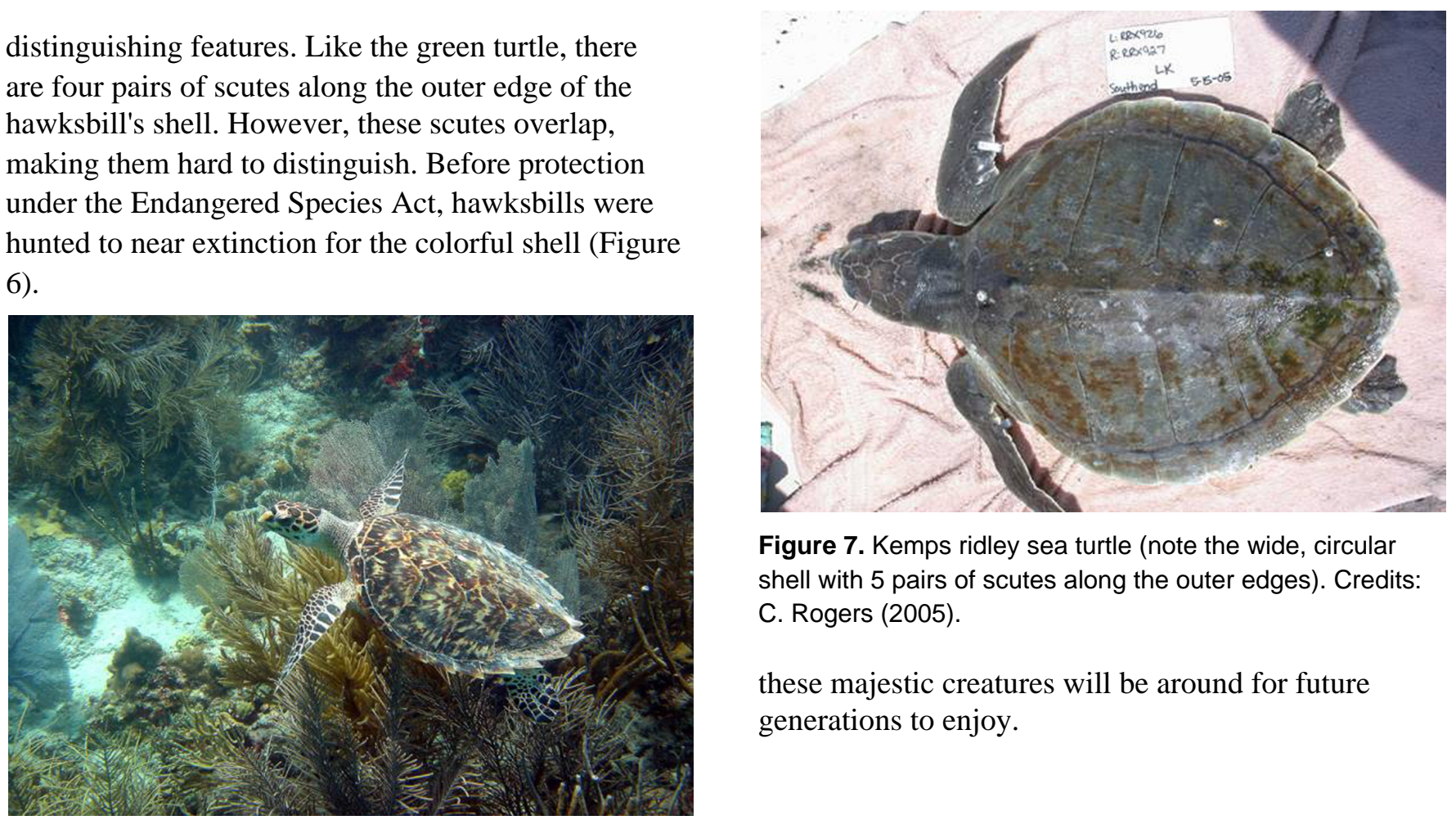

Figure 7. Kemps ridley sea turtle (note the wide, circular shell with 5 pairs of scutes along the outer edges). Credits: C. Rogers (2005).

these majestic creatures will be around for future generations to enjoy.

Figure 6. Hawksbill sea turtle (note the brightly colored shell and overlapping scutes). Credits: C. Rogers. (2005).

The Kemps ridley is the smallest of all sea turtles occurring in the United States, with an adult weight of 100 pounds. Once considered the most critically endangered sea turtle in the world, the Kemps ridley is currently making a comeback. This is a result of many years of hard work on the Kemps ridley nesting beach in Mexico and restrictions in the fishing industry. A short, wide, circular shell covers the Kemps ridley's body (Figure 7). Five pairs of scutes are typically found along the outer edge of the shell. The Kemps ridley's head is large like that of the loggerhead but less pointed. Loggerhead and Kemps ridley sea turtles have several similarities, but the Kemps ridley is much smaller and does not have a heart-shaped shell. Also, the shell of a Kemps ridley is grayish, rather than reddish-brown like a loggerhead.

\section{What You Can Do}

Due to their threatened and endangered status, the protection of sea turtles is critical for their continued survival. If you see someone entering a closed nesting area or disturbing sea turtle hatchlings or adults, call the FWC 24-hour hotline 1-888-404-3922. With your help and cooperation, 